\title{
TTR
}

Traduction, terminologie, rédaction

\section{La fabrique de la traduction, un cadrage queer : Michel Tremblay traduit par John Van Burek et Bill Glassco}

\section{Nicole Nolette et Dominique Louër}

Volume 32, numéro 2, 2e semestre 2019

La politique des microcentres : la traduction dans des contextes " mineurs » comme transfert culturel complexe

The Politics of Micro-Centers: Translation in "Minor" Contexts as Complex Cultural Transfer

URI : https://id.erudit.org/iderudit/1068907ar

DOI : https://doi.org/10.7202/1068907ar

Aller au sommaire du numéro

Éditeur(s)

Association canadienne de traductologie

ISSN

0835-8443 (imprimé)

1708-2188 (numérique)

Découvrir la revue

Citer cet article

Nolette, N. \& Louër, D. (2019). La fabrique de la traduction, un cadrage queer : Michel Tremblay traduit par John Van Burek et Bill Glassco. TTR, 32(2),

159-183. https://doi.org/10.7202/1068907ar
Résumé de l'article

Cet article porte sur la traduction vers l'anglais de la pièce de théâtre Hosanna de Michel Tremblay par John Van Burek et Bill Glassco, réalisée à Toronto au milieu des années 1970. En entrant dans la fabrique de la traduction, nous avançons l'hypothèse que, des deux éléments linguistico-culturels marquants pour les publics d'Hosanna - le joual et le sujet queer -, le second a été peu privilégié dans le travail des traducteurs. Nous abordons le carrefour entre queer studies, traduction des sociolectes et génétique de la traduction pour montrer que les traducteurs ne s'attardent pas particulièrement aux aspects queer de la pièce, mais cherchent surtout à produire, au fil des versions, une langue pour les personnages de Tremblay. 


\title{
La fabrique de la traduction, un cadrage queer: Michel Tremblay traduit par John Van Burek et Bill Glassco ${ }^{1}$
}

\author{
Nicole Nolette \\ Université de Waterloo \\ Dominique Louër \\ Université de Waterloo
}

\section{Résumé}

Cet article porte sur la traduction vers l'anglais de la pièce de théâtre Hosanna de Michel Tremblay par John Van Burek et Bill Glassco, réalisée à Toronto au milieu des années 1970. En entrant dans la fabrique de la traduction, nous avançons l'hypothèse que, des deux éléments linguistico-culturels marquants pour les publics d'Hosanna - le joual et le sujet queer -, le second a été peu privilégié dans le travail des traducteurs. Nous abordons le carrefour entre queer studies, traduction des sociolectes et génétique de la traduction pour montrer que les traducteurs ne s'attardent pas particulièrement aux aspects queer de la pièce, mais cherchent surtout à produire, au fil des versions, une langue pour les personnages de Tremblay.

Mots-clés : traduction théâtrale, génétique de la traduction, queer, joual, Canada

Abstract

This article addresses the play Hosanna by Michel Tremblay and its English translation by John Van Burek and Bill Glassco in Toronto during the 1970s. By entering the workshop of translation, we propose that of the two linguistic and cultural elements that marked the audiences of Hosanna, the Montreal sociolect joual and the queer subject matter, the second was less privileged in the work of the translators. We turn to the intersection between queer studies, translation of sociolects, and genetics of translation to show that where the translators sometimes linger on the queer aspects of the play, they mostly work, draft after draft, on constructing a language for Tremblay's characters.

Keywords: theatre translation, genetics of translation, queer, joual, Canada

1. Cette recherche a été financée par le Conseil de recherches en sciences humaines du Canada. Les auteures tiennent à remercier le Conseil de son appui ainsi que les évaluateurs anonymes de leurs précieux commentaires. 


\section{Introduction : l'héritage queer de la pièce Hosanna}

Le comédien Damien Atkins, qui tenait en 2016 le rôle du travesti Claude/Hosanna de la pièce Hosanna de Michel Tremblay dans une reprise, par la compagnie Soulpepper, de la traduction anglaise de John Van Burek et Bill Glassco, explique en ces termes l'importance symbolique de cette pièce plus de quarante ans après sa première production à Toronto en 1974 :

This play was written before I was born, and so in a way I've never known a pre-Hosanna world, right? And that may sound ridiculous but as a queer person, this is an important queer play, a very famous, early, early, queer Canadian play, and it's a play in which people speak a new world into existence, they're speaking truth about themselves and revealing things about themselves they can't go back on. And in some way this play did the same thing: it spoke a kind of truth into the world that changed the world, and I am the recipient of that change. I'm living in a world that was [...] created in some part by Hosanna and by Michel Tremblay, and by people who wrote things like this. And so, it's a great honour for me who has been living in the privilege of that for so long to go back and examine one of the things that helped create that privilege. (Soulpepper Theatre Company, 2016)

La pièce se déroule à Montréal et met en scène un coiffeur de la Plaza Saint-Hubert notoire pour son personnage de drag queen Hosanna, dont le costume imite celui d'Elizabeth Taylor dans le film américain Cléopâtre. Damien Atkins évoque le rôle déterminant de la pièce dans la construction d'un monde où il est possible de s'épanouir comme personne queer. Ce rôle, la pièce l'aura surtout exercé dans sa version anglaise, chez des spectateurs qui y voient aussi bien la représentation d'une histoire d'amour entre Claude/Hosanna et son amoureux Cuirette qu'une libération de l'identité homosexuelle (Koustas, 1989, p. 129; Dickinson, 1999, p. 111-112). Un critique du Toronto Star, par exemple, y perçoit «a classic study of homosexual revenge» (Anthony, 1974, cité dans Koustas, 1989, p. 134). Du reste, les critiques anglocanadiens ont rapidement reconnu la qualité exceptionnelle de la pièce, dont ils diront notamment qu'il s'agit de "one of the most popular plays ever to be written by a Canadian playwright» (Ossea, 1977, cité dans Koustas, 1989, p. 129).

La pièce de Michel Tremblay connaît un succès semblable auprès du public québécois francophone, mais pour des raisons différentes. L'auteur est déjà connu pour ses textes dramatiques en joual, le sociolecte de la classe ouvrière de l'est de Montréal dont les tonalités 
rappellent la double aliénation du Canada français - au pouvoir socioéconomique du Canada anglais et au pouvoir linguistique de la France - qu'il a transposé dans ses écrits destinés à la scène, au premier chef dans la pièce Les belles-søurs présentée en lecture publique en 1968. Pendant la décennie qui suit, Tremblay affine et atténue quelque peu dans sa dramaturgie le mode de transposition de cette variété linguistique. Dans la pièce Hosanna créée au Théâtre de Quat'Sous à Montréal en mai 1973, Tremblay insiste non pas sur ses personnages homosexuels, mais sur l'allégorie politique qui fait des Canadiens français des êtres travestis en quête d'authenticité : «On est un peuple qui s'est déguisé pendant des années pour ressembler à un autre peuple... On a été travestis pendant 300 ans.» (Tremblay, 1971, p. 64, cité dans Dickinson, 1999, p. 108). Autrement dit, là où on a vu le travesti Claude/Hosanna au Canada anglais, on a vu le processus analogique du travestissement au Québec (Godin et Mailhot, 1980, p. 178). Ces facteurs auront une influence marquante sur la réception de la pièce au Québec, de sorte que les spectateurs préféreront la lecture nationaliste à l'identification sur des bases (homo-)sexuelles.

Analysant le fossé entre ces deux interprétations, Peter Dickinson affirme qu'il y a là

a transnational failure in translation of Hosanna's transculturation, a refusal on the part of most anglophone critics and viewers to make the necessary link between national and sexual self-determination. (1999, p. 111 ; il souligne)

Mais cet échec transnational est-il nécessairement un échec de la traduction? Les traducteurs John Van Burek, alors directeur artistique du Théâtre du P'tit Bonheur, et Bill Glassco, alors directeur du Tarragon Theatre ${ }^{2}$, figurent parmi les premiers lecteurs anglophones et spectateurs de la pièce. Comme directeurs de théâtre, metteurs en scène et traducteurs, ils avaient pour ambition de diffuser le théâtre de Michel Tremblay à Toronto. Selon Jane Koustas (2006, p. 267), c'est à ce titre qu'ils ont transformé la pratique de la traduction au Canada. Ces traducteurs sont-ils pour autant responsables, comme le croit Dickinson, du refus généralisé de faire le pont entre affirmation nationale et genrée dans le monde anglophone? Ont-ils provoqué le malentendu autour de la valeur symbolique du travestissement du

2. Le Théâtre du P'tit Bonheur a été fondé à Toronto en 1967; il porte le nom de Théâtre français de Toronto depuis 1987. Le Tarragon Theatre a pour sa part vu le jour en 1971, à Toronto également. 
personnage de Claude/Hosanna? Nous soutenons le contraire dans le présent article. En effet, nous avançons l'hypothèse que, des deux éléments linguistico-culturels marquants pour les publics d'Hosanna - le joual et le vocabulaire queer -, le second a été peu privilégié dans le travail des traducteurs. En entrant dans la fabrique de la traduction ${ }^{3}$ grâce aux outils de la génétique textuelle, nous abordons le carrefour entre queer studies, sociolectes et traduction pour montrer que le duo de traducteurs composé de Van Burek et Glassco a travaillé activement, au fil des ébauches, à rendre la langue de Tremblay; ce faisant, les traducteurs ont amoindri certaines particularités du joual. Ce travail sur la langue de Tremblay se fait de pair avec la traduction souvent aléatoire des aspects queer de la pièce, traduction qui accentue parfois la teneur des répliques des personnages homosexuels.

\section{En amont : entre queer studies et sociolectes québécois}

Nos recherches font entrer en dialogue trois domaines qui n'ont pas souvent loccasion de se rencontrer: les queer studies, la traduction des sociolectes et l'approche génétique des textes. La polysémie du mot «queer", qui peut désigner une variété de concepts liés au genre, à la sexualité et même à la politique, se complique lorsqu'on associe ce mot à la traduction, notamment à la traduction littéraire et théâtrale. Pour William Spurlin (2015), les intrications d'une telle association relèvent de l'intraduisible, terme popularisé entre autres par Emily Apter pour désigner l'aspect subversif de l'espace entre les langues (2013, p. 61). Dans un cadrage queer, l'intraduisible remettrait en question certaines idées préconçues sur la traduction, notamment celle du simple passage qu'elle permettrait entre deux langues-cultures (Spurlin, 2015, p. 177). Ni innocente ni impuissante, la traduction se doit, selon Roland Weißegger (2001, p. 167), de transmettre les identités construites par le texte queer. En ce sens, la traduction joue fondamentalement sur le plan identitaire. Comme mode de traduction activiste (au sens où l'entend Tymoczko, 2010), la traduction queer

3. Nous utilisons l'expression fabrique de la traduction pour désigner le processus de traduction. L'expression est inspirée notamment de Fabienne Durand-Bogaert, selon laquelle «[1] a traduction est fabrique - de langues, de pensée, de textes, de littératures dites étrangères»(2014a, p. 7). Dans un dossier de la revue Genesis consacré à la génétique textuelle et publié sous la direction de Durand-Bogaert, Daria Sinichkina (2014) emploie pour sa part l'expression « fabrique des traducteurs » pour faire référence à une expérience en atelier réalisée en 2012 au Collège international de traducteurs littéraires d'Arles. Dans le cadre de celle-ci, une équipe de traducteurs a produit puis analysé des brouillons de traduction du premier chapitre du roman russe L'Arabesque d'or (1925). 
intervient à divers niveaux, le premier (et le plus évident) correspondant à des textes d'auteurs queer (Mira, 1999, p. 111) portant sur des thématiques queer et transmis dans une autre langue par le biais d'un lexique approprié (Palekar, 2017). Ce mode de traduction s'opère également autour de textes dont le sujet traduisant effectue une lecture queer, comme celle du roman Le tour du monde en quatre-vingts jours de Jules Vernes par William Butcher, qui met en œuvre une suite de choix linguistiques qui privilégient les références homosexuelles (O'Driscoll, 2008). L'inverse est aussi vrai : le processus de traduction est souvent le lieu d'un effacement (intentionnel ou non) des aspects queer, qu'ils soient explicites ou implicites, du texte littéraire. Enfin, la traduction queer peut être non pas l'œuvre des traducteurs eux-mêmes, mais celle des lecteurs d'autres polysystèmes littéraires qui découvrent les textes et les lisent de manière inattendue. Keith Harvey relève cet aspect tant dans son étude pionnière consacrée à la traduction du camp (2000a, p. 241), considéré à la fois comme une manière de parler efféminée et un comportement corporel associé aux homosexuels, que dans ses travaux ultérieurs :

translated literature occupies a special place within the space for gay readers in that translated texts can suggest models of otherness that can be used in processes of internal identity formation and imagined community projection. (2000b, p. 159)

Harvey note cependant que cet effet de traduction se concrétise principalement dans le cas où la matière du texte témoigne explicitement d'une expérience vécue de l'homosexualité et de la lutte sociale à laquelle elle donne lieu.

Les queer studies offrent une abondance de réflexions théoriques pour l'analyse de la sexualité, des identités liées au genre et des cadres non normatifs. Sur le plan méthodologique toutefois, la nature particulière de ce champ d'études continue de soulever des questions, comme le constatent Kath Browne et Catherine Nash (2016, p. 2). On peut notamment se demander quels sont les impacts des conceptualisations queer sur les choix méthodologiques, et si les méthodes appliquées en sciences sociales peuvent devenir queer (ibid.). Au Canada, Gillian Lane-Mercier émet l'hypothèse que l'une des traditions de traduction est la traduction «au féminin» et que celle-ci, après un intérêt marqué pour l'écriture féminine, a effectué un virage paradigmatique vers les "queer theories» (2014, p. 532-533). L'autre tradition de traduction au Canada, postule-t-elle, se rattache aux 
enjeux esthétiques et sociopolitiques du passage vers l'anglais du français québécois (le joual au premier chef) (ibid.).

Gillian Lane-Mercier et Catherine Leclerc ont réalisé certaines des rares études qui cernent les enjeux conjoints de ces deux traditions de traduction. Lane-Mercier (2008), reconnaissant le travail innovant de Gail Scott sur l'anglais minorisé par le français, se penche sur la mixité linguistique et la syntaxe de la traduction française du roman Heroine par Susanne de Lotbinière-Harwood. Elle soutient que bien que cette version soit célébrée par les critiques féministes pour sa reproduction de l'écriture féminine de Scott en français, la traduction "does not capture the linguistic and cultural translation effects so central to her [Scott's] experimental aesthetics» (Lane-Mercier, 2008, p. 210). La traduction en vernaculaire québécois de LotbinièreHarwood, précise-t-elle, met l'accent sur l'environnement québécois de la protagoniste et laisse oublier les malaises qu'elle ressent parfois à l'endroit de cet environnement. Dans le sillage de Lane-Mercier et largement en accord avec elle, Leclerc (2012) examine les traductions subséquentes de l'œuvre de Scott, Les Fiancées de la Main par Paule Noyart et My Paris par Julie Mazzieri. Elle constate chez Noyart une obéissance aux normes de la «bonne traduction» (Leclerc, 2012, p. 180) en langue française et à certaines des tendances déformantes qu'elles engendrent, telles que décrites par Berman : «l'effacement des superpositions de langue, la destruction des réseaux langagiers vernaculaires, l'ennoblissement et la rationalisation phrastique» (Berman, 1999, p. 49-68, cité dans Leclerc, 2012, p. 197). Ce conformisme s'étend au vocabulaire queer en traduction; par exemple, «dyke» devient «lesbienne», un choix conservateur selon Leclerc :

Le français québécois n'a pas d'équivalent du même registre pour $d y k e$, contrairement au «gouine» européen. C'est dire que les choix de traduction effectués ici sont d'abord conservateurs, que ce conservatisme soit québécois ou européen. (2012, p. 196)

Chez Mazzieri, Leclerc observe l'utilisation moins conservatrice d'un français cosmopolite marqué par de multiples ancrages géographiques. La traductrice corrige cependant les usages fautifs du français par Scott et son lexique queer. Le passage «femmes-cuir (queer), leatherwomen. Kissing» (Scott, 1999, p. 121), par exemple, est traduit comme suit par Mazzieri : "Paré pour l'occasion de femmes en cuir. Qui s'embrassent»(Scott, 2010, p. 179). Comme l'affirme Leclerc, 
[e]n omettant la pseudo-traduction, Mazzieri privilégie un lectorat francophone qui n'a pas besoin d'indications sur la prononciation du mot «cuir». Mais elle prive aussi ce lectorat de la richesse analogique de la pseudo-traduction anglaise et du vocabulaire queer à l'enseigne duquel loge une bonne part de My Paris. (2012, p. 198)

Devant un croisement semblable entre plurilinguisme littéraire et vocabulaire queer en traduction, Matthew Kayahara propose l'idée que la traduction vers l'anglais des pièces de Michel Tremblay transforme le discours joual en discours homosexuel, que les traducteurs activent le contenu gai par leurs choix de mots $(2004$, p. 83). Kayahara analyse l'intertextualité gaie, les éléments du lexique et le contenu sexuel de trois pièces de Tremblay en français - Hosanna, Les anciennes odeurs et Messe solennelle pour une pleine lune d'été - en comparant ces aspects dans la traduction en anglais pour conclure que les changements observés ne sont pas assez importants pour soutenir son hypothèse. Pour Kayahara, dans le cas de ces trois pièces, le processus de traduction ne serait donc pas le lieu d'une lecture queer de la version française. Reste néanmoins, comme le souligne Renate Usmiani, que les traductions vers l'anglais des pièces de Tremblay partagent plusieurs insuffisances, notamment une réduction de la variété des jurons au profit du mot «shit», l'effacement de la superposition de langues présent dans le joual ainsi qu'un conservatisme certain par rapport aux expressions sexuelles (1982, p. 27-28). Vivien Bosley parle pour sa part d'une «dilution» du joual par les traducteurs John Van Burek et Bill Glassco, «as it is standardized into generic North American» (1988, p. 140141). Louise Ladouceur ajoute au sujet de la traduction anglaise de la pièce Hosanna que Van Burek et Glassco préconisent le recours aux gallicismes, emprunts et calques, mais que ceux-ci n'apparaissent que dans les répliques du personnage de Claude/Hosanna et qu'ils disparaissent dans la réédition de 1991 (2005, p. 92). Nous retiendrons des analyses de Kayahara, Usmiani, Bosley et Ladouceur qu'elles mettent en évidence une histoire des retraductions contraire à celle de Gail Scott. Dans le cas de John Van Burek et Bill Glassco traduisant et retraduisant Michel Tremblay, devant la double difficulté des sociolectes et du vocabulaire queer, le conservatisme des traducteurs s'accroît. La traduction confirme les tendances déformantes décrites par Berman et mises à profit par Leclerc, notamment celles de l'effacement des superpositions des langues et de la destruction des réseaux langagiers vernaculaires. 


\section{En amont : la génétique de la traduction théâtrale}

Les textes qui, tels ceux mentionnés à la section précédente, commentent le joual ou les questions de genre en traduction font habituellement référence aux traductions une fois qu'elles ont été publiées, ou au produit fini de la traduction. L'approche de la génétique des textes, dont l'application aux traductions est relativement récente (Bourjea, 1995; Durand-Bogaert, 2014a; Cordingley et Montini, 2015; Montini, 2016), permet de pousser la recherche plus loin. La génétique des textes «enquête sur la fabrique, débusque les processus, remonte le cours de l'œuvre, scrute les traces» (Durand-Bogaert, 2014a, p. 7). Dans le numéro de la revue Genesis qu'elle a dirigé sur la traduction, Fabienne Durand-Bogaert explique qu'il reste rarement des traces du processus de traduction, que ni les maisons d'édition ni les bibliothèques ne montrent beaucoup d'intérêt pour les archives de traducteurs et que les traducteurs eux-mêmes hésitent à partager leurs brouillons et cahiers à moins d'avoir participé à une entreprise collective (Durand-Bogaert, 2014b, p. 16-17). À partir d'archives de traducteurs qu'elle a trouvées, Durand-Bogaert formule tout de même quelques hypothèses sur le processus :

Si la phase «prétraductive» au cours de laquelle le traducteur réunit de la documentation, annote son original ou procède à l'examen de ce que sa lecture a déposé en lui peut se comparer à la phase prérédactionnelle repérable chez l'écrivain, la suite du processus tend à se singulariser. (ibid., p. 28)

La phrase rédactionnelle du travail des traducteurs diffère de celle des écrivains en ce qu'elle commence rarement par un plan, qu'il soit détaillé ou non, mais le plus souvent par un premier brouillon lancé rapidement sur la page pour ensuite être révisé, raturé, retravaillé, ou alors rédigé très lentement au rythme du polissage, puis très peu retouché par la suite. Parfois, les ratures et modifications présentes dans les brouillons montrent un retour au texte de départ, dans un "mécanisme de feed-back herméneutique» (Ladmiral cité par Durand-Bogaert, 2014b, p. 30) qui informe la version finale. Sous toutes ces formes, les brouillons de traduction contiennent de multiples annotations d'opérations qui intéressent les généticiens du texte - ajouts, suppressions, déplacements et remplacements - malgré l'hypothèse de Durand-Bogaert voulant que la suppression soit la moins importante des opérations en ce qui concerne les traducteurs. 
Bien que la génétique des traductions théâtrales ait fait l'objet de très peu de recherches jusqu'à présent (voir, par exemple, Vasserot, 2016), du côté des études théâtrales, Josette Féral $(1998$; 2008) et, à sa suite, Almuth Grésillon, Marie-Madeleine Mervant-Roux et Dominique Budor (2010), ont jeté les bases d'une génétique de la performance ou de l'événement théâtral qui prend en compte les carnets de mise en scène, les captations vidéo et les notes prises durant l'observation des répétitions. De notre côté, nous avons bénéficié pour notre étude des avant-textes de traduction conservés aux archives de la bibliothèque McLaughlin de l'Université de Guelph, dans les dossiers du Théâtre du P'tit Bonheur/Théâtre français de Toronto. Ces avanttextes sont attribués à John Van Burek et Bill Glassco, quoiqu'il n'y ait souvent qu'une seule main à l'œuvre. Certains dossiers contiennent plusieurs ébauches de traductions d'une même œuvre tandis que d'autres dossiers incluent une seule traduction accompagnée de la version finale de la pièce en version tapuscrite ou publiée.

Le dossier de la pièce Hosanna (1973), présentée en anglais au Tarragon Theatre en 1974, contient quatre versions; c'est grâce à celles-ci que nous avons pu examiner les traces matérielles de la traduction. Les deux premières versions se suivent dans un cahier noir de 201 pages numérotées à gauche. Ce sont des versions manuscrites. $\mathrm{La}$ troisième version, qu'on trouve dans un cahier rouge de 56 pages, est dactylographiée et contient des notes écrites à la main au crayon ou au stylo; elle est décrite comme un "prompt script» dans le catalogue des archives. Dans la pratique théâtrale, le prompt script est un outil de travail utilisé pour la régie, la mise en scène et le jeu des acteurs pendant les répétitions d'un spectacle. Dans le cas de la traduction d'Hosanna, le prompt script est un signe important des préoccupations scéniques des traducteurs (Koustas, 1989, p. 132). La quatrième version de la pièce apparaît dans des épreuves dactylographiées de la publication chez Talonbooks en 1974; elle est donc destinée aux lecteurs plutôt qu'aux spectateurs. Cette version fait 178 pages; on y trouve quelques commentaires annotés à la main ainsi que des ratures.

Après avoir numérisé ces quatre versions de la traduction pour mieux les visualiser, nous avons produit les transcriptions diplomatiques ${ }^{4}$ observables dans les exemples que nous présentons plus loin. Nous avons ensuite identifié les ajouts, suppressions, déplacements

4. Une transcription diplomatique " "photographie" le document en rapportant, avec les outils qui le permettent, malgré leurs limites, tous les événements du manuscrit» (Fekete et Crasson, n. d., n. p.). 
et remplacements dans chacune des versions avant de les comparer. Comme Kahayara (2004) dans son analyse du queer dans les traductions anglaises des pièces de Tremblay, notre attention s'est d'abord portée sur les endroits où ces opérations prenaient pour objet un élément du lexique queer ou des pronoms associant le personnage de Claude/Hosanna aux genres féminin, masculin ou non binaire. Dans les sections qui suivent, nous examinons ces aspects ainsi que les mots ou structures associés au joual.

\section{Hosanna et la traduction aléatoire du queer}

Dans son analyse de la traduction d'Hosanna par Van Burek et Glassco, Kayahara (2004) montre que le traitement fluctuant des éléments du lexique et des pronoms pourrait amplifier la lecture queer de la pièce. Notre étude des avant-textes confirme cette assertion. Dans le texte original de Tremblay, malgré l'ambivalence associée au personnage de Claude/Hosanna, le pronom et les adjectifs autoréférentiels sont toujours accordés au féminin. Dans les didascalies de la traduction anglaise, le pronom féminin demeure: "Elle se regarde dans le miroir» (Tremblay, 1973a, p. 13) devient ainsi «She looks into the mirror» (Tremblay, 1973b, p. 2). La traduction investit davantage le féminin dans les répliques des personnages : "C'est un travesti cheap» (Tremblay, 1973a, p. 13) est traduit par "She is a cheap transvestite» (Tremblay, 1973b, p. 2). Certaines insultes taquines lancées en français par les deux personnages dans leur dialogue conflictuel, et pourtant peu liées au genre féminin, sont également orientées vers le féminin dans la traduction. C'est ce qu'on peut voir dans le tableau 1.

Tableau 1. Exemples de traductions orientées vers le féminin selon Kayahara (2004, p. 94)

\begin{tabular}{|l|l|}
\hline Hosanna (Tremblay, 1973a) & Hosanna (Tremblay, 1973b) \\
\hline maudite putain sale (p. 19) & you dried up cunt (p. 18) \\
\hline maudite niaiseuse (p. 36) & you stupid twat (p. 50) \\
\hline maudite kétaine (p. 13) & stupid bitch (p. 10) \\
\hline
\end{tabular}

La traduction du dernier exemple reproduit au tableau 1 («stupid bitch») se trouve dans les cahiers de Van Burek dès le premier jet, même si les traducteurs suppriment puis remplacent le mot «stupid» à quelques reprises. Kayahaha avance que le mot «bitch ", quant à lui, revient dans la traduction anglaise comme équivalent de «vache» et de 
«bitch»en français, amplifiant ainsi la culture de la «bitchiness» (ou de la vacherie!) de la drag queen mise en scène par le spectacle (2004, p. 94). On remarque par contre dans les extraits reproduits au tableau 2 que, dans l'avant-texte, ce sont les adjectifs et non les substantifs qui font l'objet de remplacements.

Tableau 2. Exemples de «bitchiness» selon Kayahara (2004, p. 94) dans l'avant-texte

\begin{tabular}{|l|l|}
\hline \multicolumn{1}{|c|}{ Hosanna (Tremblay, 1973a) } & Hosanna (Tremblay, 1973b) \\
\hline $\begin{array}{l}\text { Maudite quétaine! Maudite } \\
\text { quétaine! Maudite quétaine... } \\
\text { (p. 13) }\end{array}$ & $\begin{array}{l}\text { You stupid bitch! You stupid } \\
\text { stupid bitch bitch. Stupid You } \\
\text { stupid, stupid bitch! (p. 3) }\end{array}$ \\
\hline $\begin{array}{l}\text { t'une des plus grandes folles de } \\
\text { Montréal (p. 40) }\end{array}$ & $\begin{array}{l}\text { One of the biggest best known } \\
\text { biggest queens of Montreal... } \\
\text { (p. 44) }\end{array}$ \\
\hline
\end{tabular}

En fait, les mots qui relèvent explicitement d'un vocabulaire queer provoquent rarement des changements dans les avant-textes, où ils ne sont pas associés à des ratures, des déplacements ou des remplacements. L'expression "de même», par contre, fait l'objet de plusieurs opérations. Dans Hosanna, Tremblay utilise cette expression pour évoquer de manière floue les pratiques homosexuelles et efféminées de ses personnages. Ces allusions sont rendues de différentes façons dans la traduction. On remarque d'abord une hésitation; deux traductions sont proposées : "like this" et "the way I am» (première ligne du tableau 3, page suivante). La traduction est plus claire ailleurs dans le premier brouillon, où le mot «gay» est inscrit au stylo noir sans que d'autres options immédiates soient données pour traduire «de même». Plus loin dans le même brouillon, le mot «queer» est utilisé, puis supprimé, ce qui montre un certain flottement dans le lexique.

La dernière ligne du tableau 3 correspond à une transcription provenant du cahier noir du premier jet de la traduction. Dans le passage tel qu'il se présente dans le cahier, on voit pour la première fois le travail de trois stylos de couleurs différentes, bleu pour le texte initial, rouge et noir pour les modifications subséquentes. Le "she told» en bleu est raturé en bleu, et il y a une deuxième tentative en bleu, "my mother", raturée en rouge pour être remplacée par «she», aussi en rouge au-dessus de "my mother». Le «told me» en bleu, dont la rature est de la même couleur, est remplacé par «said» en bleu dans la 
marge de gauche après «told». Un X noir vient couper le «that» bleu. Le «been» bleu est remplacé par «started» en rouge placé au-dessus du mot raturé trois fois en rouge. Le «said» en bleu fait l'objet d'une autre version superposée en rouge, «says", de nouveau remplacée par «said» de la même couleur au-dessus du mot. Une grande rature en bleu traverse "If you're gonna be a queer Claude». Une flèche noire à gauche pointe vers la marge où il est écrit et gribouillé ce qui pourrait être (nous n'en sommes pas certaines) «itd» en noir. La page miroir contient cependant d'autres versions, une en noir «it doesn't matter," avec une rature rouge, et une en bleu ("If that's the way you want to be, Claude». Une première version en bleu de «at least pick out the good looking ones» est raturée en noir ("at least») et en rouge ("piek out the»), puis remplacée par «make sure they're» surimposé en rouge au-dessus de "piek out the». "Just» est ajouté en noir au-dessus du texte en bleu, tout de suite avant "piek». Le mot «ones», quant à lui, disparaît sous une rature rouge.

Tableau 3. Traduction de l'expression «de même»

\begin{tabular}{|l|l|}
\hline Hosanna (Tremblay, 1973a) & Hosanna (Tremblay, 1973b) \\
\hline $\begin{array}{l}\text { Ma mère, a'le sait pas que chus de } \\
\text { même, pis... (p. 38) }\end{array}$ & $\begin{array}{l}\text { My mother doesn't know I'm like- } \\
\text { this, and... the way I am, and... } \\
\text { (p. 39). }\end{array}$ \\
\hline $\begin{array}{l}\text { Tu sais c'qu'àm'a dit, ma mère, } \\
\text { quand àl'a appris que j'étais de } \\
\text { même, hein? (p. 41) }\end{array}$ & $\begin{array}{l}\text { You know want to know what } \\
\text { my mother said when she found } \\
\text { out I was gay? Hutr?You want to } \\
\text { know? (p. 44). }\end{array}$ \\
\hline $\begin{array}{l}\text { Ben, sais-tu c'qu'à m'a répondu, } \\
\text { ma mère, quand j’y ai dit que } \\
\text { j'avais commencé à coucher avec } \\
\text { les hommes? A m'a dit : Si t'es de } \\
\text { même, mon p'tit gars, au moins, } \\
\text { choisis-toé-s'en des beaux! (p. 42) }\end{array}$ & $\begin{array}{l}\text { You know what she told said my } \\
\text { mother told me when I told her } \\
\text { men? She said says said: If youtre- } \\
\text { gonna be a queer Claude, at least } \\
\text { good looking ones! (p. 46). }\end{array}$ \\
\hline
\end{tabular}

Dans les autres versions, la phrase "If that's the way you want to be» est conservée. Par l'usage du mot "queer» dans une première tentative de traduction de ce passage, le texte revisite un mot utilisé précédemment pour traduire le québécisme «tapette» (Tremblay, 
1973a, p. 41). On observe cependant un flottement, car «tapette» est aussi traduit par «fag» ailleurs dans le texte. Dans la traduction du passage concernant la mère de Claude (dernière ligne du tableau 3), le mot «queer» est cependant vite remplacé par une phrase qui est plus près de la version initiale : "She said, "If that's the way you want to be, Claude, just make sure they're good looking”" (Tremblay, 1973b,p. 45). Cette retraduction dans le premier brouillon illustre particulièrement bien l'efficacité du mécanisme de «feed-back herméneutique» décrit par Ladmiral (cité dans Durand-Bogaert, 2014b, p. 30), où le retour sur le texte de départ signale le rapprochement avec le texte d'arrivée.

De manière similaire au traitement souvent aléatoire du vocabulaire queer, le travestissement, présent dans l'ensemble du texte de Tremblay, est parfois nommé "drag" dans le texte source et parfois évoqué par l'expression "s'habiller en femme». Dans les deux cas, le mot "drag» est utilisé dans la traduction quoique, dans le deuxième cas, il s'agit d'un ajout qui est rapidement remplacé par l'expression préalable «dressed up like women», plus littérale.

Tableau 4. Traduction de l'expression «s'habiller en femme»

\begin{tabular}{|l|l|}
\hline Hosanna (Tremblay, 1973a, p. 30) & $\begin{array}{l}\text { Hosanna (Tremblay, 1973b, p. 10- } \\
\text { 11) }\end{array}$ \\
\hline $\begin{array}{l}\text { les chauffeurs de taxi qui se } \\
\text { promènent habillés en femmes } \\
\text { se font plutôt rares... Sont plutôt } \\
\text { rares, les chauffeurs de taxi } \\
\text { habillés en femme! }\end{array}$ & $\begin{array}{l}\text { Taxi drivers who go around } \\
\text { dressed up like like women are pretty rare... } \\
\end{array}$ \\
$\begin{array}{l}\text { In fact they're very rare, taxi } \\
\text { drivers dressed tu like } \\
\text { drag. taxi drivers dressed up like } \\
\text { women! }\end{array}$ \\
\hline
\end{tabular}

Ce que le cahier noir ne dit pas dans ce cas, c'est l'explicitation du travestissement qui se met en place, puis qui se voit supprimée en deux opérations consécutives.

Enfin, si les choix des traducteurs semblent souvent aléatoires ou redevables au mécanisme de feedback herméneutique, on peut cependant affirmer que la traduction amplifie parfois au fil des opérations les références et l'humour queer du texte de Tremblay. C'est le cas dans la réplique humoristique du personnage d'Hosanna observable au tableau 5 (page suivante). 
Tableau 5. Suites d'opérations pour la traduction de «folle»

\begin{tabular}{|l|l|}
\hline $\begin{array}{l}\text { Hosanna (Tremblay, } \\
\text { 1973a, p. 40) }\end{array}$ & $\begin{array}{l}\text { Chus pas une des plus grandes folles de } \\
\text { Montréal, pis t'es gêné de parler de ça avec ta } \\
\text { mère... }\end{array}$ \\
\hline $\begin{array}{l}\text { Hosanna, version 1 } \\
\text { (Tremblay, 1973b, } \\
\text { p. 44) }\end{array}$ & $\begin{array}{l}\text { f'm not one of the biggest queens. I think } \\
\text { we've realized tonight that I'm hardly not one } \\
\text { of the biggest queens in town, Montreal and as } \\
\text { for my mother, I'm not "embarrassed." to talk }\end{array}$ \\
\hline $\begin{array}{l}\text { Hosanna, version 2 } \\
\text { (Tremblay, 1973b, } \\
\text { p. 44) }\end{array}$ & $\begin{array}{l}\text { After what happened tonight, I hardly think } \\
\text { we know ean say that I'm not one of the } \\
\text { biggest queens... }\end{array}$ \\
\hline $\begin{array}{l}\text { Hosanna, version 3 } \\
\text { (Tremblay, 1973b, } \\
\text { p. 44) }\end{array}$ & $\begin{array}{l}\text { On the contrary, lover boy. One of the biggest } \\
\text { queens, what are you, blind! After tonight, I } \\
\text { think I'm one of the smallest. And as for my } \\
\text { mother, I'm not afraid to talk to her about } \\
\text { anything... }\end{array}$ \\
\hline $\begin{array}{l}\text { Hosanna, version 4 } \\
\text { (Tremblay, 1973b, } \\
\text { p. 176 [p. 40]) }\end{array}$ & $\begin{array}{l}\text { Not anymore I'm not, not after tonight. As for } \\
\text { my old lady, I'm not afraid to talk to her about } \\
\text { anything my treasure. }\end{array}$ \\
\hline $\begin{array}{l}\text { Hosanna, version 5 } \\
\text { (Tremblay, 1973c, } \\
\text { p. 25) }\end{array}$ & $\begin{array}{l}\text { One of the biggest queens, are you blind? Not } \\
\text { after tonight, I'm not. As for my old lady, my } \\
\text { treasure, I'm not afraid to talk to her about } \\
\text { anything... }\end{array}$ \\
\hline $\begin{array}{l}\text { Hosanna, version 6 } \\
\text { (Tremblay, 1974, } \\
\text { p. 26) }\end{array}$ & $\begin{array}{l}\text { One of the biggest queens, are you blind? Not } \\
\text { after tonight, I'm not. As for my old lady, my } \\
\text { treasure, I'm not afraid to talk to her about } \\
\text { anything... }\end{array}$ \\
\hline
\end{tabular}

Dans la version 1 reproduite ci-dessus, Van Burek et Glassco proposent une première traduction assez conventionnelle au stylo noir, puis remplacent une phrase traduite littéralement par une expression plus idiomatique. La traduction se précise: le mot "hardly" est remplacé par «not», "town" par "Montreal». Une flèche à gauche suggère une autre version de la même réplique (version 2 ci-dessus), dans laquelle "hardly» apparaît en bleu foncé au-dessus de «think». Dans le même cahier, les traducteurs effectuent une deuxième version 
complète (version 3), dans laquelle le mot "gêné», d'abord rendu par «embarassed», est ensuite traduit par «not afraid». Le passage "One of the biggest queens, what are you, blind!», écrit au crayon, est remplacé par la version 4, dans laquelle apparaissent les expressions «old lady» et "my treasure». Les deux autres dossiers (versions 5 et 6) offrent le même texte, sans annotations. Les expressions ajoutées dans les dernières versions - "lover boy" (version 3), "old lady» et «my treasure» (version 4) - ajoutent une couche de performance du féminin toute en camp à la parole du personnage d'Hosanna en anglais. En somme, malgré les traductions souvent aléatoires des aspects queer de la pièce, les traducteurs accentuent parfois le camp des répliques du personnage de la drag queen.

\section{Hosanna sous les ratures de la traduction du joual}

On a vu dans les exemples qui précèdent que le vocabulaire queer est le lieu de peu d'opérations dans le texte. En fait, ce qui transparaît dans les ratures de diverses couleurs et les ajouts et remplacements subséquents, c'est un travail sur l'oralité et la langue populaire. La traduction de "A'm'a dit», par exemple, alterne entre «She said", "She says" et "She said», alors que "y'a» et "y'en a» deviennent "You've got», "there are», "there'sre» et "There's Iguress there's a». D'autres exemples montrent que cette tendance se maintient. Les extraits reproduits au tableau 6 illustrent la façon dont la réplique «Sont comme pardus...» est traduite (Tremblay, 1973a, p. 22).

Tableau 6. La traduction de "pardus"

\begin{tabular}{|l|l|}
\hline $\begin{array}{l}\text { Hosanna (Tremblay, 1973a, } \\
\text { p. 22) }\end{array}$ & Sont comme pardus... \\
\hline $\begin{array}{l}\text { Hosanna, version 1 } \\
\text { (Tremblay, 1973b, p. 7) }\end{array}$ & they're eompletely lost done for... \\
\hline $\begin{array}{l}\text { Hosanna, version 2 } \\
\text { (Tremblay, 1973b, p. 131) }\end{array}$ & $\begin{array}{l}\text { They're lost. It's sort of like they're } \\
\text { lost... }\end{array}$ \\
\hline $\begin{array}{l}\text { Hosanna, version } 3 \\
\text { (Tremblay, 1973c, p. 10) }\end{array}$ & It's sort of like they're lost... \\
\hline $\begin{array}{l}\text { Hosanna, version 4 } \\
\text { (Tremblay, 1974, p. 10) }\end{array}$ & It's sort of like they're lost... \\
\hline
\end{tabular}


Si les premières versions sont retravaillées plusieurs fois, les deux dernières, destinées respectivement à la scène et à la publication, ne portent aucune modification ou annotation. Comme on peut le voir au tableau 7, c'est aussi le cas d'une autre réplique marquée par l'écriture de l'oralité, dont la syntaxe est retravaillée dans les deux premières versions. Les traducteurs remplacent aussi «mama " par «maman», ce qui est cohérent avec le désir de conserver les noms propres dans la langue de départ. Une rature du "we» dans la version vouée à la scène la rapproche de la version publiée.

\section{Tableau 7. La traduction de «moman»}

\begin{tabular}{|l|l|}
\hline $\begin{array}{l}\text { Hosanna } \text { (Tremblay, } \\
\text { 1973a, p. 25) }\end{array}$ & $\begin{array}{l}\text { C'est ça, fait c'que ta moman t'a montré de } \\
\text { faire... }\end{array}$ \\
\hline $\begin{array}{l}\text { Hosanna, version 1 } \\
\text { (Tremblay, 1973b, p. 12) }\end{array}$ & $\begin{array}{l}\text { That's it, take mama's adviee, do like } \\
\text { mama showed you. }\end{array}$ \\
\hline $\begin{array}{l}\text { Hosanna, version 2 } \\
\text { (Tremblay, 1973c, p. 12) }\end{array}$ & That's right, do it like Maman said. \\
\hline $\begin{array}{l}\text { Hosanna, version 3 } \\
\text { (Tremblay, 1974, p. 12) }\end{array}$ & That's right, we do it like Maman said. \\
\hline
\end{tabular}

L'enjeu pratique de la traduction du joual est d'autant plus visible dans les tirades du personnage d'Hosanna, ce qu'on voit au tableau 8. Dans la première version de la traduction, le mot "parsonne» devient «nobody" ou «no one», mais «travarse» demeure «take a ride». Dans ce passage, la rature sur "Lafontaine» est au crayon, les autres sont au stylo bleu, comme l'écriture initiale. Le crayon se pose sur la page pour ajouter quelques mots : «right», "while you're at it», «the», «about», "I've broken» et «one». Ces changements délocalisent le texte; l'action pourrait maintenant se dérouler dans n'importe quel parc et dans n'importe quelle ville. Plus loin dans le même cahier, tout en conservant la délocalisation, les traducteurs se reprennent et produisent la version 2 . On voit qu'ils tentent de rétablir la structure de la phrase française ("Have a good») mais y renoncent finalement, et qu'ils uniformisent l'emploi des jurons («Goddamn it! Shit»). Curieusement, cette version contient aussi un ajout au crayon dans la marge - «struggling with dress» - qui pourrait agir comme une didascalie ou un rappel pour le régisseur ou les acteurs. Ni l'une ni l'autre des deux versions subséquentes, destinées respectivement à la 
scène et à la publication, ne contiennent d'autres modifications ou annotations.

Tableau 8. Traduction du joual et des jurons, et délocalisation du texte

\begin{tabular}{|l|l|}
\hline $\begin{array}{l}\text { Hosanna (Tremblay, } \\
\text { 1973a, p. 22) }\end{array}$ & $\begin{array}{l}\text { C'est ça, vas-y donc, pis travarse donc le Parc } \\
\text { Lafontaine en passant... Va donc pleurer sur } \\
\text { tes débuts qui intéressent parsonne pis que } \\
\text { t'achales tout le monde avec... Cibole, j'me } \\
\text { sus cassé un ongle! Verrat de bâtard, c'est le } \\
\text { deuxième à soir! }\end{array}$ \\
\hline $\begin{array}{l}\text { Hosanna, version 1 } \\
\text { (Tremblay, 1973b, } \\
\text { p. 6) }\end{array}$ & $\begin{array}{l}\text { Go right ahead! And while you're at it, take a } \\
\text { ride through the Parc Eafontaine white youtre- } \\
\text { at it... You can stop and have a good cry over } \\
\text { your tragic past, about which nobody no one } \\
\text { could care less, about but with which you } \\
\text { tore everyone to tears... Goddamn it, I broke } \\
\text { I've broken a nail! That's the second one time } \\
\text { tonight! Shit! }\end{array}$ \\
\hline $\begin{array}{l}\text { Hosanna, version 2 } \\
\text { (Tremblay, 1973b, } \\
\text { p. 125) }\end{array}$ & $\begin{array}{l}\text { Go right ahead! And while you're at it, take a } \\
\text { ride through the park. Have a good You can } \\
\text { stop and have a good cry over the scene of your } \\
\text { début, which no one gives a shit about, even } \\
\text { though you love to bore everyone to death } \\
\text { with it... Goddann it! Shit! I've broken a nail! } \\
\text { That's the second one tonight! Shit! }\end{array}$ \\
\hline
\end{tabular}

Enfin, un dernier exemple montre une incertitude quant au sens de certains mots propres au joual comme "pogner", observable dans une autre tirade du personnage d'Hosanna reproduite au tableau 9 (page suivante). La première traduction dans le cahier noir propose deux options : "They $\mathrm{He}$ still go for him grabs them! etc. They $\mathrm{He}$ grabs em allright still go for him! In the dark, yeah! In the meat racks, yeah!» (Tremblay, 1973b, p. 51). Le stylo rouge introduit une rature qui traverse le texte rédigé en noir et le remplace par «He grabs em allright». Entre deux sens vernaculaires, celui de séduire et celui d'attraper, Van Burek choisit, à tort ou à raison, le second. De plus, en intégrant "meat-racks» comme tel, il efface la superposition des langues présente dans l'original. La deuxième version dans ce même cahier entérine ces choix, mais introduit un emprunt, "ouais», qui 
rétablit une certaine forme de superposition des langues dans les répliques du personnage d'Hosanna. Dans cette nouvelle forme de superposition, le français ne signale pas la présence du joual (l'original utilisait le mot "oui»); il reflète plutôt le style camp qui amplifie l'aspect queer du personnage d'Hosanna. Les deux versions qui suivent (versions 3 et 4 ci-dessous) confirment l'écriture de l'oralité et l'emprunt: dans la version 3 (destinée à la scène), une barre oblique est ajoutée au milieu de «all/right», de même que deux «Ouais» au stylo bleu. Ces deux modifications sont respectées dans la version publiée (version 4), avec toutefois une minuscule au mot «ouais». Cet exemple consolide ce qu'illustraient déjà les exemples précédents, à savoir le questionnement des traducteurs et leurs multiples opérations autour du joual, le travail sur l'oralité du joual en traduction, la substitution de la superposition des langues par une amplification de l'aspect camp des répliques du personnage d'Hosanna.

Tableau 9. «Pogner» et la traduction du joual

\begin{tabular}{|l|l|}
\hline $\begin{array}{l}\text { Hosanna } \text { (Tremblay, } \\
\text { 1973a, p. 44) }\end{array}$ & $\begin{array}{l}\text { Y pogne encore! Dans'noirceur, oui! Dans } \\
\text { les meat-racks, oui! }\end{array}$ \\
\hline $\begin{array}{l}\text { Hosanna, version 1 } \\
\text { (Tremblay, 1973b, } \\
\text { p. 51) }\end{array}$ & $\begin{array}{l}\text { They He still go for him grabs them! etc. } \\
\text { They He grabs em allright still go for hint In } \\
\text { the dark, yeah! In the meat racks, yeah! }\end{array}$ \\
\hline $\begin{array}{l}\text { Hosanna, version 2 } \\
\text { (Tremblay, 1973b, } \\
\text { p. 44-45) }\end{array}$ & $\begin{array}{l}\text { He still grabs them! He grabs them, allright! } \\
\text { In the dark, yeah-ouais! In the meat-racks, } \\
\text { yeahrouais! }\end{array}$ \\
\hline $\begin{array}{l}\text { Hosanna, version 3 } \\
\text { (Tremblay, 1973c, } \\
\text { p. 28) }\end{array}$ & $\begin{array}{l}\text { He still grabs them! He still grabs them all/ } \\
\text { right! In the dark, Ouais! In the meat-racks, } \\
\text { Ouais! }\end{array}$ \\
\hline $\begin{array}{l}\text { Hosanna, version 4 } \\
\text { (Tremblay, 1974, p. 52) }\end{array}$ & $\begin{array}{l}\text { He still grabs them! He still grabs them all } \\
\text { right! In the dark, ouais! In the meat-racks, } \\
\text { ouais! }\end{array}$ \\
\hline
\end{tabular}

\section{Hosanna, entre le queer et le joual}

Il est possible de croiser dans d'autres exemples les deux aspects, le queer et le joual, pour voir Van Burek et Glassco à l'œuvre. C'est le cas dans la réplique reproduite au tableau 10. 
Tableau 10. La traduction du queer et du joual (1)

\begin{tabular}{|l|l|}
\hline $\begin{array}{l}\text { Hosanna (Tremblay, } \\
\text { 1973a, p. 68-69) }\end{array}$ & $\begin{array}{l}\text { Gang d'hosties, d'écourants, vous avez toute } \\
\text { changé, hein, vous avez toute changé! Maudits } \\
\text { chiens sales! [...] Y'a autant de tapettes } \\
\text { dans'police qu'y'n a ailleurs! Pis y'a autant de } \\
\text { tapettes dans les gars de bicycle qu'y'en a ailleurs, } \\
\text { j'ai l'honneur de vous apprendre! }\end{array}$ \\
\hline $\begin{array}{l}\text { Hosanna, version 1 } \\
\text { (Tremblay, 1973b, } \\
\text { p. 13) }\end{array}$ & $\begin{array}{l}\text { You filthy shit-faced bastards, you've gone } \\
\text { and changed it all around the whole place, eh, } \\
\text { you've changed the whole place it all around! Ya } \\
\text { pigs! [...] You've got as many fags in the poliee } \\
\text { department as there are-anywhere else. And } \\
\text { maybe there are just as lots of many fags who } \\
\text { are eops as there are bikers. Maybe Maybe there } \\
\text { are a lots of fags who ride bikes, but I have the } \\
\text { pleasure of informing you that there are just as } \\
\text { many fags in the police department! }\end{array}$ \\
\hline $\begin{array}{l}\text { Hosanna, version 2 } \\
\text { (Tremblay, 1973b, } \\
\text { p. 14) }\end{array}$ & $\begin{array}{l}\text { And If there'sre a lot of fags who ride bikes. } \\
\text { There's Iguess there's a just as many fags tots- } \\
\text { of fags in the fucking police... department, eh? } \\
\text { And that's a fact! }\end{array}$ \\
\hline $\begin{array}{l}\text { Hosanna, version 3 } \\
\text { (Tremblay, 1973c, } \\
\text { p. 42) }\end{array}$ & $\begin{array}{l}\text { You filthy bastards, you're gone and changed it } \\
\text { around, eh, you've changed it all around! You Ya } \\
\text { pigs! [...] And if there's lots of fags who ride } \\
\text { bikes, there's just as many fags in the fuckin' } \\
\text { police force, and that's a fact! }\end{array}$ \\
\hline
\end{tabular}

Des ratures noires s'imposent sur un premier jet de traduction, et on trouve un premier remplacement, «it all around", pour "the whole place", toujours en noir, après "changed». Entre "but» et "there» s'ajoute le segment «I have the pleasure of informing you that». Une autre version au crayon (version 2) se trouve sur la page miroir de la première version. L'emploi du mot «fag" $\mathrm{y}$ pose moins problème et engendre moins d'opérations que la syntaxe oralisée du joual ("You've got/Maybe Maybe there are»), le temps de verbe («there's/there're») et les jurons de la réplique («fucking» est ajouté en bleu). Dans la version dactylographiée de la production (version 3), c'est toujours le problème du degré d'oralité qui se pose. Au-dessus de "Ya pigs", 
les traducteurs écrivent «You» au crayon. Ils retirent «shit-faced» et ajoutent "fuckin", transforment "department» en "force», ce qui donne la version 3 reproduite au tableau 10 .

La même situation s'observe dans l'extrait reproduit au tableau 11, où Hosanna exprime dans un monologue son rapport au travestissement et à l'emploi. Dans le cahier noir, les traducteurs proposent d'abord une version en bleu et quelques commentaires au crayon. Les ratures sont au stylo bleu, sauf pour celles de "I'm dressed». $\mathrm{Au}$-dessus de «they'tld drop me» figure la note au crayon «That'll be the end of", alors que sur la page de gauche est ajouté au stylo bleu «Sure. I'll be right out of a job.». Dans cette première version, la syntaxe oscille pour s'éloigner de la langue française, les coiffures des «Juives jewish-renaissance» devenant d'énigmatiques "JewishRenaissance permanents to the ladies». Pour rendre la réplique qui était en anglais et en italique dans le texte original ("You should work in drag, Claude!»), des guillemets sont utilisés en anglais. Par contre, on observe une hésitation quant au temps de verbe («they'td drop me on the spot»).

\section{Tableau 11. Traduction du monologue d'Hosanna}

\begin{tabular}{|l|l|}
\hline $\begin{array}{l}\text { Hosanna } \\
\text { 1973a, p. 29) }\end{array}$ & $\begin{array}{l}\text { Chus ridicule quand chus déguisée en homme, quand } \\
\text { j'coiffe mes Juives jewish-renaissance. Des vrais gestes } \\
\text { de femmes, qu'y me disent que j'ai... You should work } \\
\text { in drag, Claude! Pis si j'irais travailler en femme j'gage } \\
\text { qu'y me laisseraient tomber parce qu'y veulent pas se } \\
\text { laisser toucher aux cheveux par des femmes... Pis chus } \\
\text { ridicule quand chus déguisée en femme parce que } \\
\text { j't'obligée de faire la folle pour attirer l'attention parce } \\
\text { que chus pas assez belle pour l'attirer autrement... Pis } \\
\text { chus t'encore plus ridicule quand chus pognée comme } \\
\text { ça, entre les deux, avec ma tête de femme, mes sous- } \\
\text { vêtements de femme, pis mon corps... }\end{array}$ \\
\hline $\begin{array}{l}\text { Hosanna, } \\
\text { version 1 } \\
\text { (Tremblay, } \\
\text { 1973b, p. 40) }\end{array}$ & $\begin{array}{l}\text { F'm ridieutous When I'm dressed like a man, I'm } \\
\text { ridiculous giving Jewish-Renaissance permanents to } \\
\text { the ladies. "Very feminine gestures, they tell me... "You } \\
\text { should be working in drag, Claude!" Hath! The day I go } \\
\text { to work in drag, they'Hd drop me on the spotbecause } \\
\text { they can't stand having their hair touched by another } \\
\text { woman... }\end{array}$ \\
\hline
\end{tabular}




\begin{tabular}{|c|c|}
\hline $\begin{array}{l}\text { Hosanna, } \\
\text { version } 2 \\
\text { (Tremblay, } \\
\text { 1973b, } \\
\text { p. } 19-20 \text { ) }\end{array}$ & 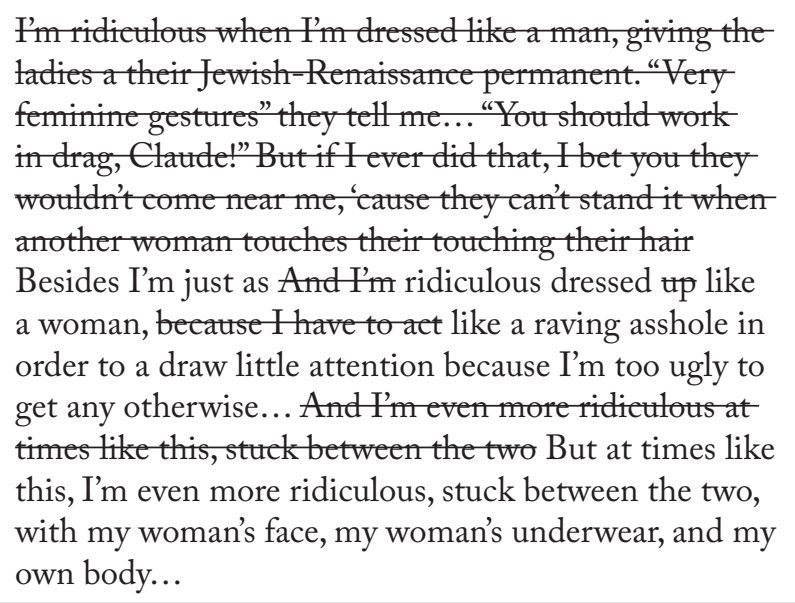 \\
\hline $\begin{array}{l}\text { Hosanna, } \\
\text { version } 3 \\
\text { (Tremblay, } \\
\text { 1973c, p. 15) }\end{array}$ & 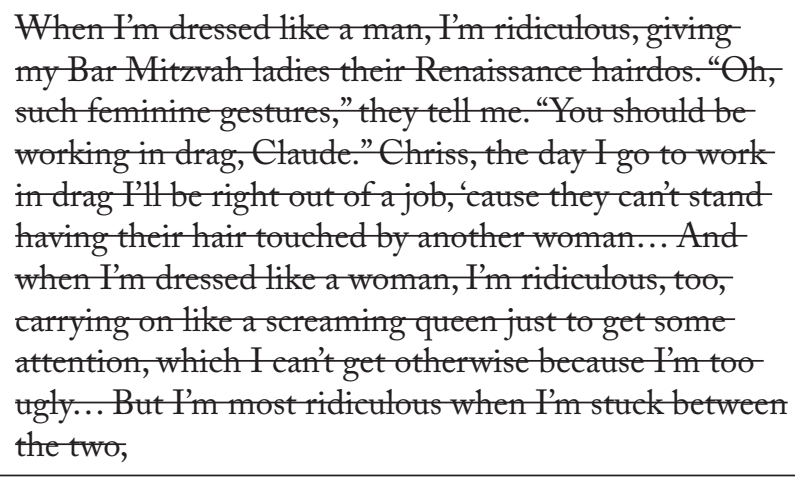 \\
\hline $\begin{array}{l}\text { Hosanna, } \\
\text { version } 4 \\
\text { (Tremblay, } \\
\text { 1973c, p. 16) }\end{array}$ & 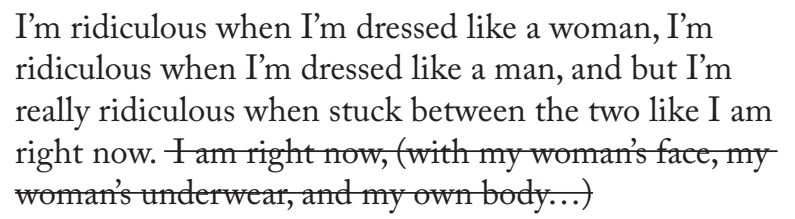 \\
\hline
\end{tabular}

La deuxième version dans le cahier noir (version 2 ci-dessus) montre d'autres hésitations par rapport à la syntaxe parlée du texte. Dans le cahier rouge (version 3 ci-dessus), ces hésitations aboutissent à un gros « $\mathrm{X}$ » en noir qui traverse la version dactylographiée de la traduction de l'extrait. On voyait déjà entre ces deux cas des transformations du segment "Jewish-Renaissance permanents to the ladies" en "giving my Bar Mitzvah ladies their Renaissance hairdos", et le mot «Chriss» ajouté là où il n'y avait pas de juron en français. Au crayon, 
à la gauche de cet extrait biffé, un gros point d'interrogation s'impose avec l'annotation "ugly». Un autre "x», plus petit, marque la marge de droite. Sur la page blanche au recto, les traducteurs font une autre tentative qui se poursuit sur la page dactylographiée suivante (version 4 $\mathrm{du}$ tableau 11). Cette dernière tentative est suivie du commentaire «keep in", aussi en rouge. L'annotation "ugly» nous hante toutefois : quelle manière de communiquer la frustration du traducteur devant la complexité à rendre le passage en joual! Son choix sera de faire disparaître "pis chus» ou "And when I'm» pour mettre le sujet («I», «my») à l'avant-plan de la phrase. L'économie - plus précisément la concision - guide cette approche qui fait d'un registre neutre, oralisé et accentué de jurons la solution d'un processus ardu de traduction du joual.

\section{Conclusion : l'héritage de la traduction d'Hosanna}

Les cahiers de John Van Burek et Bill Glassco, on le voit, contiennent maintes traces du processus de traduction qui montrent la gestion en cours d'une affirmation croisée sur les plans sexuel et nationaliste. Les stratégies de traduction utilisées, qui paraissent diversifiées et aléatoires dans les deux cas, témoignent toutefois d'une tentative plus forte de réponse à la question de la traduction du joual par rapport à celle du queer. Les opérations se multiplient, les remplacements sont fréquents. Comme l'indique Gillian Lane-Mercier, c'est au cours des années 1970, alors même que Van Burek et Glassco travaillent à leur traduction de la pièce Hosanna, que la question de la traduction du joual se joue et se décide :

il y a fort à parier que, à force d'être mobilisées en fonction d'impératifs de plus en plus consensuels liés à l'apparition au sein de la culture cible (le Canada anglais) de normes et d'attentes fondées, notamment, sur la lisibilité et l'acceptabilité, ces stratégies se sont «normalisées» pour donner lieu, dans le courant des années 1970 et 1980, à ce qui pourrait ressembler à une tradition de traduction de la québécitude ou, plus modestement, du joual. (2014, p. 531; elle souligne)

Dans leur traduction d'Hosanna, Van Burek et Glassco participent à la construction de ces normes et attentes fondées sur l'acceptabilité : ils construisent une langue orale neutre, marquée pour le personnage travesti de quelques éléments camp. Du point de vue de la génétique de la traduction, l'héritage d'Hosanna touche à ces opérations où le commentaire "ugly» transparaît implicitement ou apparaît explicitement, et des versions acceptables se manifestent. En ce sens, il n'est 
pas clair qu'on puisse parler, comme le fait Dickinson (1999), d'un échec de traduction, ni d'ailleurs d'un refus de faire le pont entre affirmation nationale et sexuée. L'héritage traductologique d'Hosanna reste paradoxal, dans la mesure où, par sa réception, la représentation de la pièce en anglais a eu des répercussions importantes sur la communauté queer de Toronto et du Canada anglais. En amont, dans la fabrique de la traduction, se trouvent cependant deux traducteurs torontois et leur recherche à tâtons, à coup de ratures et de remplacements, de solutions visant à représenter le joual ou du moins l'oralité et, dans une moindre mesure, l'affirmation sexuée du personnage de Claude/Hosanna.

\section{Références}

Apter, Emily (2013). Against World Literature: On the Politics of Untranslatability, New York, Verso.

Bosley, Vivien (1988). «Diluting the Mixture: Translating Michel Tremblay's Les Belles-soeurs». TTR, 1, 1, p. 139-145.

Bourjea, Serge, dir. (1995). Génétique Ė traduction. Paris, L'Harmattan.

Browne, Kath et Catherine J. Nash (2016). Queer Methods and Methodologies: An Introduction. Londres et New York, Routledge.

Cordingley, Anthony et Chiara Montini (2015). «Genetic Translation Studies: An Emerging Discipline». Linguistica Antverpiensia, New Series - Themes in Translation Studies, 14. [https://lans-tts.uantwerpen.be/index.php/LANSTTS/article/view/399].

Dickinson, Peter (1999). Here is Queer: Nationalisms, Sexualities, and the Literatures of Canada. Toronto, University of Toronto Press.

Durand-Bogaert, Fabienne (2014a). «Ce que la génétique dit, la traduction le fait.» Genesis. Manuscrits - Recherche - Invention, 38, p. 7-10.

Durand-Bogaert, Fabienne (2014b). «Les deux corps du texte». Genesis. Manuscrits - Recherche - Invention, 38, p. 11-33.

Féral, Josette (1998). «Pour une analyse génétique de la mise en scène». Théâtre/ Public, 144, p. 54-59.

Féral, Josette (2008). «Introduction: Towards a Genetic Study of Performance - Take $2 »$. Theatre Research International, 33, 3, p. 223-233.

Fekete, Jean-Daniel et Aurèle Crasson (n. d.). «Structuration des manuscrits : $\mathrm{du}$ corpus à la région». ITEM : Institut des textes et manuscrits modernes. [http://www.item.ens.fr/articles-en-ligne/structuration-des-manuscritsdu-corpus-a-la-region/].

Godin, Jean-Cléo et Laurent Mailhot (1980). Théâtre québécois II. Nouveaux auteurs, autres spectacles. Montréal, Hurtubise HMH.

Grésillon, Almuth, Marie-Madeleine Mervant-Roux et Dominique Budor, dir. (2010). Genèses théâtrales. Paris, CNRS.

Harvey, Keith (2000a). «Describing Camp Talk: Language/Pragmatics/ Politics». Language and Literature: International Journal of Stylistics, 9, 3, p. 240-260. 
Harvey, Keith (2000b). «Gay Community, Gay Identity and the Translated Text». TTR, 13, 1, p. 137-165.

Kayahara, Matthew (2004). "Travestis Politiques": The Gay Value of Michel Tremblay's Dramatic Work in Translation. Thèse de maîtrise. École de traduction et d'interprétation, Université d'Ottawa. Inédit.

Koustas, Jane (1989). "Hosanna in Toronto: "Tour de force" or "Détour de traduction"?». TTR, 2, 2, p. 129-139.

Koustas, Jane (2006). «John Van Burek: Bringing Tremblay to Toronto». In A. Whitfield, dir. Writing between the Lines: Portraits of Canadian Anglophone Translators. Waterloo, Wilfrid Laurier University Press, p. 267-286.

Ladouceur, Louise (2005). Making the Scene. La traduction du théâtre d'une langue officielle à l'autre au Canada. Québec, Nota Bene.

Lane-Mercier, Gillian (2008). «From Minor to Minority: Susanne de Lotbinière-Harwood Translating Gail Scott Translating "My Montréal"». In D. Merkle et al., dir. Traduire depuis les marges/Translating from the Margins. Québec, Nota Bene, p. 193-227.

Lane-Mercier, Gillian (2014). «Les carences de la traduction littéraire au Canada : des bibliographies et des traditions ». Meta, 59, 3, p. 517-536.

Leclerc, Catherine (2012). «Whose Paris (and whose Montréal)? Gail Scott en français et la littérature québécoise». Revue d'études canadiennes, 46, 3, p. $170-203$.

Mira, Alberto (1999). «Pushing the Limits of Faithfulness: A Case for Gay Translation». In J. Boase-Beier et M. Holman, dir. The Practices of Literary Translation: Constraints and Creativity. Manchester, St. Jerome, p. 109-123. Montini, Chiara, dir. (2016). Traduire: genèse du choix. Paris, Éditions des archives contemporaines.

O'Driscoll, Kieran (2008). "Around the World in Eighty Gays: Retranslating Jules Verne from a Queer Perspective». In D. De Crom, dir. Translation and the (Trans)formation of Identities. Selected Papers of the CETRA Research Seminar in Translation Studies. Louvain, CETRA, p. 1-31.

Palekar, Shalmalee (2017). «Remapping Translation: Queering the Crossroads ». In B.J. Epstein et R. Gillett, dir. Queer in Translation. Londres et New York, Routledge, p. 8-24.

Sinichkina, Daria (2014). «L'expérience de la Fabrique des Traducteurs : les brouillons de traduction comme espace de confrontation et de réappropriation ». Genesis. Manuscrits - Recherche - Invention, 38, p. 99-109.

Scott, Gail (1999). My Paris: A Novel, Toronto, Mercury Press.

Scott, Gail (2010). My Paris. Trad. Julie Mazzieri. Montréal, Héliotrope.

Soulpepper Theatre Company (2016). «The Legacy and Lineage of Hosanna ». [Vidéo]. [https://www.youtube.com/watch?time_continue $=1608 v=v 9 \mathrm{G}$ $\mathrm{KiHPVy} 4 \mathrm{k}]$.

Spurlin, William (2015). "Queering Translation: Rethinking Gender and Sexual Politics in the Spaces between Languages and Cultures». In B.J. Epstein et R. Gillett, Queer in Translation. Londres et New York, Routledge, p. 172-183. 
Tremblay, Michel (1973a). Hosanna. Suivi de La duchesse de Langeais. Montréal, Leméac.

Tremblay, Michel (1973b). Hosanna. Trad. John Van Burek et Bill Glassco. Cahier noir, University of Guelph Library Guelph McLaughlin Archives - Théâtre du P'tit Bonheur/Théâtre Français, Guelph. XZ1 MS A551018.

Tremblay, Michel (1973c). Hosanna. Trad. John Van Burek et Bill Glassco. Cahier rouge, University of Guelph Library Guelph McLaughlin Archives - Théâtre du P'tit Bonheur/Théâtre Français, Guelph. XZ1 MS A551018. Tremblay, Michel (1974). Hosanna. Trad. John Van Burek et Bill Glassco. Vancouver, Talonbooks. University of Guelph Library Guelph McLaughlin Archives - Théâtre du P'tit Bonheur/Théâtre Français, Guelph. XZ1 MS A551018.

Tymoczko, Maria, dir. (2010). Translation, Resistance, Activism. Amherst, University of Massachusetts Press.

Usmiani, Renate (1982). Michel Tremblay. Vancouver, Douglas \& McIntyre.

Vasserot, Christilla (2016). «Traduction théâtrale et surtitrage : la remise en cause des choix. Le cas d'Angélica Liddel». In C. Montini, dir. Traduire: genèse du choix. Paris, Éditions des archives contemporaines, p. 103-11.

Weißegger, Roland (2001). «Queering Translation: Transcultural Communication and the Site of You». Graduate Journal of Social Science, 8, 2, p. 164178.

Nicole Nolette

Département d'études françaises

Université de Waterloo Waterloo (Ontario), Canada nicole.nolette@uwaterloo.ca

Dominique Louër Département de psychologie Université de Waterloo Waterloo (Ontario), Canada dlouer@uwaterloo.ca 